\title{
Concurrent chemoradiotherapy improves survival outcome in muscle-invasive bladder cancer
}

\author{
Sang Jun Byun, MD', Jin Hee Kim, MD, PhD², Young Kee Oh, PhD², Byung Hoon Kim, MD³ \\ ${ }^{1}$ Yongsan Health Subcenter, Jangheung; Departments of ${ }^{2}$ Radiation Oncology and ${ }^{3}$ Urology, Dongsan Medical Center, \\ Keimyung University School of Medicine, Daegu, Korea
}

Purpose: To evaluate survival rates and prognostic factors related to treatment outcomes after bladder preserving therapy including transurethral resection of bladder tumor, radiotherapy (RT) with or without concurrent chemotherapy in bladder cancer with a curative intent.

Materials and Methods: We retrospectively studied 50 bladder cancer patients treated with bladder-preserving therapy at Keimyung University Dongsan Medical Center from January 1999 to December 2010. Age ranged from 46 to 89 years (median, 71.5 years). Bladder cancer was the American Joint Committee on Cancer (AJCC) stage II III, and IV in 9, 27, and 14 patients, respectively. Thirty patients were treated with concurrent chemoradiotherapy (CCRT) and 20 patients with RT alone. Nine patients received chemotherapy prior to CCRT or RT alone. Radiation was delivered with a four-field box technique (median, $63 \mathrm{~Gy}$; range, 48.6 to $70.2 \mathrm{~Gy})$. The follow-up periods ranged from 2 to 169 months (median, 34 months).

Results: Thirty patients (60\%) showed complete response and $13(26 \%)$ a partial response. All patients could have their own bladder preserved. Five-year overall survival (OS) rate was 37.2\%, and the 5-year disease-free survival (DFS) rate was 30.2\%. In multivariate analysis, tumor grade and CCRT were statistically significant in OS.

Conclusion: Tumor grade was a significant prognostic factor related to OS. CCRT is also considered to improve survival outcomes. Further multi-institutional studies are needed to elucidate the impact of RT in bladder cancer.

Keywords: Urinary bladder neoplasm, Bladder preservation, Concurrent chemoradiotherapy, Radiotherapy, Survival rate, Prognostic factor

\section{Introduction}

Muscle-invasive bladder cancer (MIBC) constitutes about 30\% of newly diagnosed bladder cancers, with about 70\% being non-invasive. About 15\% of non-invasive bladder cancer cases progress to invasive cancer after transurethral resection of bladder tumor (TURBT) [1,2].

Radical cystectomy has been a curative treatment option for MIBC. However, mortality and morbidity rates after surgery cannot be ignored [3]. For many patients who are unfit for surgery due to old age, poor medical condition, or patient refusal, radiotherapy (RT) is a good alternative therapeutic option. Surgery produces a 5-year local control rate of about $50 \%$ and overall survival (OS) rates of $20 \%$ to $40 \%$ [4-8]. More recently, bladder-preserving therapy including maximal TURBT followed by RT with or without concurrent chemotherapy in

Received 26 August 2015, Revised 21 September 2015, Accepted 22 October 2015.

Correspondence: Jin Hee Kim, MD, PhD, Department of Radiation Oncology, Dongsan Medical Center, Keimyung University School of Medicine, 56 Dalseong-ro, Jung-gu, Daegu 41931, Korea. Tel: +82-53-250-7665, Fax: +82-53-250-7984, E-mail: jhkim@dsmc.or.kr

(c) This is an Open Access article distributed under the terms of the Creative Commons Attribution Non-Commercial License (http://creativecommons.org/ licenses/by-nc/4.0/) which permits unrestricted non-commercial use, distribution, and reproduction in any medium, provided the original work is properly cited.

www.e-roj.org 
selected patients has become widely used.

Bladder-preserving therapy has several benefits compared to radical surgery. Patients with bladder-preserving therapy can have a better quality of life with intact bladder function [2]. In several recent studies, survival outcomes after bladderpreserving therapy are reportedly comparable to those seen in radical cystectomy series [9-14].

The present study was designed to retrospectively examine survival rates and to evaluate prognostic factors related to treatment outcomes after bladder-preserving therapy with TURBT, RT, and chemotherapy.

\section{Materials and Methods}

We analyzed 50 patients with biopsy-proven, the American Joint Committee on Cancer (AJCC) stage II-IV bladder cancer who underwent bladder-preserving therapy at Keimyung University Dongsan Medical Center from January 1999 to December 2010. In the same period, there were 572 patients who were diagnosed to have bladder cancer in our institution, regardless of AJCC stage. The study was approved by the Institutional Review Board of Keimyung University Dongsan Medical Center, and informed consent was waived.

Patients underwent physical examinations, complete blood count $(\mathrm{CBC})$, liver function tests, urinalysis, chest radiography, cystoscopy with biopsy, and pelvic magnetic resonance imaging (MRI) or computed tomography (CT) before treatments. Patient performance status was evaluated according to the guidelines of the Eastern Cooperative Oncology Group (ECOG) [15]. During RT, CBC was checked at least once a week. When the absolute neutrophil count was $<1,000 / \mathrm{mm}^{3}$ or the platelet count $<50,000 / \mathrm{mm}^{3}$, treatments were interrupted or delayed until the patient's condition recovered. Red blood cell transfusion was given to patients with hemoglobin levels below $10.0 \mathrm{~g} / \mathrm{dL}$.

TURBT was performed to get accurate diagnosis and maximal tumor removal. If needed, repeated TURBT was also done during follow-up in some patients with suspicion of recurrence. Most underwent TURBT under spinal anesthesia.

External beam RT (EBRT) was delivered using 6, 10, 15, or $20 \mathrm{MV}$ photon beams with a four-field box technique to a dose of 63 Gy in 35 fractions for 5 days per week within 7 weeks. To define the initial pelvic fields in most of the patients, the superior border was at the middle of the sacroiliac joint or at the L5-S1 interspace and the interior border was at or just below the bottom of the obturator foramen. The inferior border was sometimes extended to the bottom of the ischial tuberosity, depending on disease involvement with the prostatic urethra or bladder neck. The lateral border was 1.5 $\mathrm{cm}$ lateral to the true pelvis to encompass the bladder and the pelvic lymph nodes. On the lateral portal, the anterior border was placed in front of the bladder and the posterior border was set with at least a $3-\mathrm{cm}$ margin behind the posterior border bladder wall. The portals were reduced after $45 \mathrm{~Gy}$ (1.8 Gy per fraction) with a follow-up imaging study using a CT scan and the boost treatment included an initial gross tumor volume with 2-cm margin.

Concurrent chemotherapy was administered every week by intravenous infusion. Cisplatin $\left(40 \mathrm{mg} / \mathrm{m}^{2}\right)$ was given on the first day of the chemotherapy cycle (D1, D8, D15, and D22) within 16 hours after RT.

After completion of treatment, all patients were evaluated by radiation oncologists and urologic oncologist at 3-month intervals for 1 year, and at least 6 months thereafter. Complete response (CR) was defined as no evidence of gross tumor on follow-up cystoscopic examination, and pelvic MRI or CT 3 months after completion of treatment. Partial response (PR) was defined as $>30 \%$ reduction of gross tumor volume compared to the initial tumor [16]

Local recurrence was defined as any disease recurrence lesions within pelvic radiation fields that had developed 3 months later, after confirmation of CR to treatment. Distant metastasis was also defined as any disease outside the pelvic radiation fields.

OS time was calculated from the time of diagnosis to the date of death or last follow-up. Disease-free survival (DFS) was calculated from the end of primary treatment to the date of diagnosing locoregional recurrence or distant metastasis in patients with CR after RT. Kaplan-Meier analysis was used to estimate the OS and DFS rates, with the log-rank test for prognostic significance, and Cox proportional hazards model for multivariate analysis. A chi-square test was used to analyze the relation between factors in the two groups. A p-value less than 0.05 was considered statistically significant. Statistical analyses were performed using IBM SPSS ver. 21.0 (IBM, Armonk, NY, USA).

\section{Results}

The pretreatment characteristics of the 50 patients are listed in Table 1. Patient age ranged from 46 to 89 years with a median of 71.5 years. Gross hematuria was the most common initial symptom in 37 patients. Seven patients suffered from dysuria, one patient complained increased urinary frequency, and the 
Table 1. Patient characteristics

\begin{tabular}{|c|c|}
\hline Variable & No. $(\%)$ \\
\hline Age (yr), median (range) & $71.5(46-89)$ \\
\hline \multicolumn{2}{|l|}{ Sex } \\
\hline Male & $33(66)$ \\
\hline Female & $17(34)$ \\
\hline \multicolumn{2}{|l|}{ ECOG performance status } \\
\hline 0 & $44(88)$ \\
\hline 1 & $5(10)$ \\
\hline 2 & $1(2)$ \\
\hline \multicolumn{2}{|l|}{ Initial symptom } \\
\hline Hematuria & $37(74)$ \\
\hline Dysuria & 7 (14) \\
\hline Incidental finding & $5(10)$ \\
\hline Frequent voiding & $1(2)$ \\
\hline \multicolumn{2}{|l|}{ Cell type } \\
\hline Urothelial carcinoma & $49(98)$ \\
\hline Squamous cell carcinoma & $1(2)$ \\
\hline \multicolumn{2}{|l|}{ Tumor grade } \\
\hline High & $33(66)$ \\
\hline Low & $12(24)$ \\
\hline Unknown & $5(10)$ \\
\hline \multicolumn{2}{|l|}{ Multiplicity } \\
\hline Solitary & $31(62)$ \\
\hline Multiple & $16(32)$ \\
\hline Diffuse & $2(4)$ \\
\hline Unknown & $1(2)$ \\
\hline \multicolumn{2}{|l|}{ Clinical T stage } \\
\hline 2 & $10(20)$ \\
\hline 3 & $30(60)$ \\
\hline 4 & $10(20)$ \\
\hline \multicolumn{2}{|l|}{ Clinical N stage } \\
\hline 0 & $37(74)$ \\
\hline 1 & $10(20)$ \\
\hline 2 & $2(4)$ \\
\hline 3 & $1(2)$ \\
\hline \multicolumn{2}{|l|}{ Clinical TNM stage } \\
\hline$\|$ & $9(18)$ \\
\hline III & $27(54)$ \\
\hline IV & $14(28)$ \\
\hline \multicolumn{2}{|l|}{ Hydronephrosis } \\
\hline Present & $19(38)$ \\
\hline Absent & $31(62)$ \\
\hline
\end{tabular}

ECOG, Eastern Cooperative Oncology Group.

rest five patients were diagnosed incidentally. Patients had several underlying diseases, such as angina pectoris or acute myocardial infarction in five patients, hypertension in 15 , and diabetes mellitus in nine. Fourteen patients had personal history of cigarette smoking.

Most patients showed good performance status of ECOG 0 or 1. On pathologic examination, 49 patients (98\%) had urothelial
Table 2. Treatment variables

\begin{tabular}{lc}
\hline \multicolumn{1}{c}{ Variable } & Value \\
\hline Operation & \\
TURBT & $47(94)$ \\
Partial cystectomy & $1(2)$ \\
No & $2(4)$ \\
No. of TURBT & $3(6)$ \\
0 & $39(78)$ \\
1 & $8(16)$ \\
$\geq 2$ & $9(18)$ \\
Neoadjuvant chemotherapy & $41(82)$ \\
Yes & \\
No & $30(60)$ \\
Radiotherapy & $20(40)$ \\
CCRT & $63(48.6-70.2)$ \\
RT alone & $35(27-39)$ \\
EBRT dose (Gy) & $51(41-74)$ \\
EBRT fractions & \\
EBRT days & \\
Concurrent chemotherapy & Cisplatin \\
Regimen & $3(1-8)$ \\
Cycle(s)
\end{tabular}

Values are presented as number (\%) or median (range).

TURBT, transurethral resection of bladder tumor; CCRT, concurrent chemotherapy and radiotherapy; RT, radiotherapy; EBRT, external beam radiotherapy.

carcinoma and one (2\%) had squamous cell carcinoma. About two-thirds (33 patients) had high grade tumors. AJCC stage was II in nine patients (18\%), III in 27 patients (54\%), and IV in 14 patients (28\%). Thirty-one patients (62\%) showed solitary tumor in bladder, 16 patients (32\%) had multiple tumors, and two patients (4\%) with diffuse appearing tumor in CT scan. Hydronephrosis was reported in 19 patients (38\%) and 31 patients (62\%) had normal kidneys. Initial hemoglobin level ranged from 8.6 to $14.9 \mathrm{~g} / \mathrm{dL}$ with a median of $11.15 \mathrm{~g} / \mathrm{dL}$.

Treatment details are summarized in Table 2. Forty-seven patients (94\%) underwent TURBT for accurate pathologic diagnosis and maximal tumor eradication, and one patient (2\%) had partial cystectomy. The remaining two patients were diagnosed using cystoscopic examination and cytology due to their poor medical conditions. Of the 47 patients with TURBT, 39 patients underwent TURBT once, six patients did twice. The remaining two patients continued repeated TURBT, three times and five times, respectively. Complete TURBT was done in 11 patients and incomplete TURBT in 36 patients (Table 3).

After pathologic diagnosis, nine patients (18\%) had systemic chemotherapy before RT. Chemotherapeutic regimens were GC (gemcitabine and cisplatin) or MVAC (methotrexate, 
vinblastine, doxorubicin, and cisplatin) for 2 to 10 cycles (median, 8 cycles). Three patients showed PR after systemic chemotherapy, two patients remained in stable disease, and the rest four patients showed disease progression.

Thirty patients (60\%) had RT combined with synchronous chemotherapy using cisplatin regimen with 1 to 8 cycles (median, 3 cycles). Other 20 patients (40\%) were treated with RT alone. The range of EBRT was 48.6 to $70.2 \mathrm{~Gy}$ (median, 63 Gy) and it took 41 to 74 days (median, 51 days) to finish RT.

Three months after completion of treatments, 30 patients (60\%) showed CR and 13 (26\%) displayed PR. Five patients

Table 3. Tumor response and failure pattern

\begin{tabular}{lc}
\hline \multicolumn{1}{c}{ Variable } & No. (\%) \\
\hline Completeness of TURBT & $11(23.4)$ \\
Complete & $36(76.6)$ \\
Incomplete & \\
Tumor response after RT & $30(60)$ \\
Complete response & $13(26)$ \\
Partial response & $5(10)$ \\
Progression & $2(4)$ \\
Stable disease & $12(24)$ \\
Recurrence & $38(76)$ \\
Yes & \\
No & $9(18)$ \\
Metastasis & $41(82)$ \\
Yes & \\
No & $12(24)$ \\
Status & $1(2)$ \\
No evidence of disease & $6(12)$ \\
Alive with disease & $31(62)$ \\
Intercurrent death & \\
Died of disease &
\end{tabular}

TURBT, transurethral resection of bladder tumor; RT, radiotherapy.

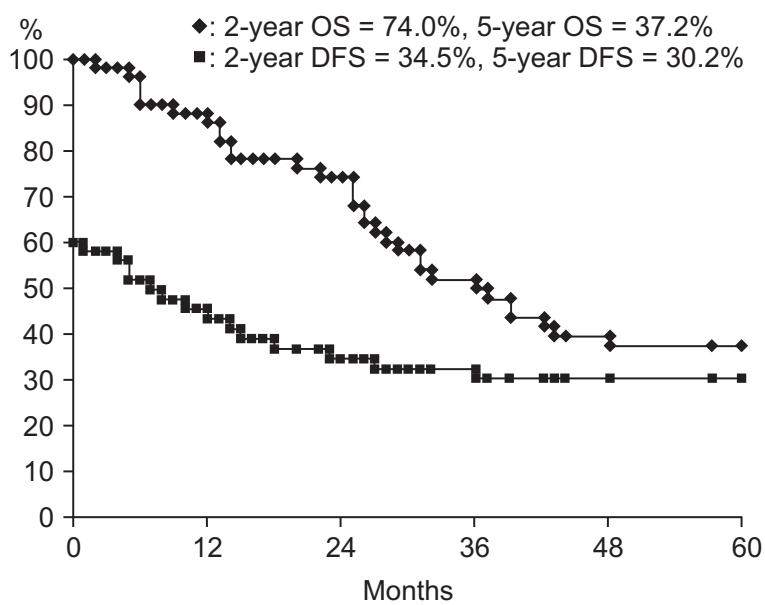

Fig. 1. Overall survival (OS) and disease-free survival (DFS) curves. showed progressed disease and the rest two remained without response to RT. Concerning patient status, 12 patients had no evidence of disease, one were alive with disease, six died from other causes, and 31 died from bladder cancer (Table 3).

The range of follow-up was 2 to 169 months (median, 34 months) in all patients. Two-year and 5-year OS rate was $74.0 \%$ and $37.2 \%$, respectively, and 2-year and 5-year DFS rate was $34.5 \%$ and $30.2 \%$, respectively (Fig. 1). During the followup period, 10 patients had local recurrence, seven patients had distant metastasis, and two patients had both local recurrence and distant metastasis (Table 3). The specific sites of local recurrence were within the bladder (11 patients) and sigmoid colon (one patient). Of the nine patients with distant metastases, four showed multiple bone metastases including skull and spine, four in the lung, and one in both multiple bones and lung. There was no patient who underwent salvage cystectomy during the follow-up time.

On univariate analysis, concurrent chemotherapy (CCRT vs. RT alone; $p=0.043$ ) was a significant prognostic factor in OS (Fig. 2). Tumor grade (low vs. high; $p=0.051$ ), clinical T stage (cT2 vs. cT3 vs. cT4; $p=0.089$ ), and clinical $N$ stage (cN negative vs. $\mathrm{cN}$ positive; $p=0.087$ ) showed marginal significance in OS. OS curves according to tumor grade are in Fig. 3. Clinical $N$ stage ( $c N$ negative vs. $c N$ positive; $p=$ 0.014 ) and clinical TNM stage (II vs. III vs. IV; $p=0.015$ ) were statistically significant prognostic factors in DFS (Fig. 4). These factors are listed in Table 4. Completion of TURBT, number of TURBT, and neoadjuvant chemotherapy were not prognostic

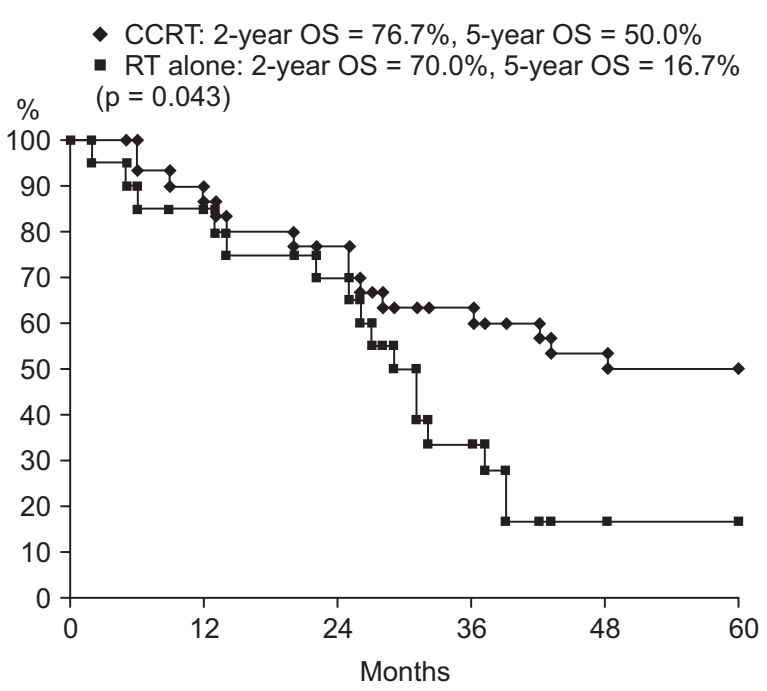

Fig. 2. Overall survival (OS) curves according to concurrent chemotherapy. CCRT, concurrent chemotherapy and radiotherapy; $R T$, radiotherapy. 
factors related to survival rates.

On multivariate analysis, among the prognostic factors with $p \leq 0.1$ on univariate analysis, tumor grade (hazard radio [HR], 2.334; 95\% confidence interval [CI], 1.007 to 5.408) and concurrent chemotherapy $(\mathrm{HR}, 0.442 ; 95 \% \mathrm{Cl}, 0.213$ to 0.915$)$ were statistically significant for OS (Table 5). According to the chi-square test, there was no statistically significant difference in the relation between factors such as completion of TURBT, number of TURBT, and clinical stage in the two groups, respectively (low grade vs. high grade, CCRT vs. RT alone).

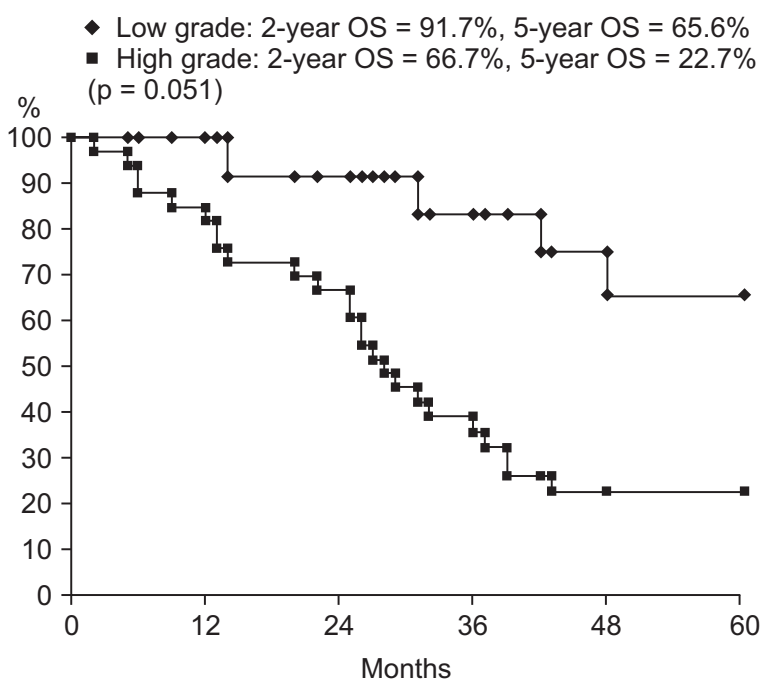

Fig. 3. Overall survival (OS) curves according to tumor grade.

\section{Discussion and Conclusion}

Radical cystectomy still remains the standard curative treatment in MIBC. Recently, bladder-preserving therapy with maximal TURBT, RT, and concurrent chemotherapy has been evolved [2]. Our study was designed to evaluate survival rates and prognostic factors related to treatment outcomes after bladder-preserving therapy including RT in MIBC. There were several limitations to this single institutional retrospective study. First, it is not feasible to generalize our results due to

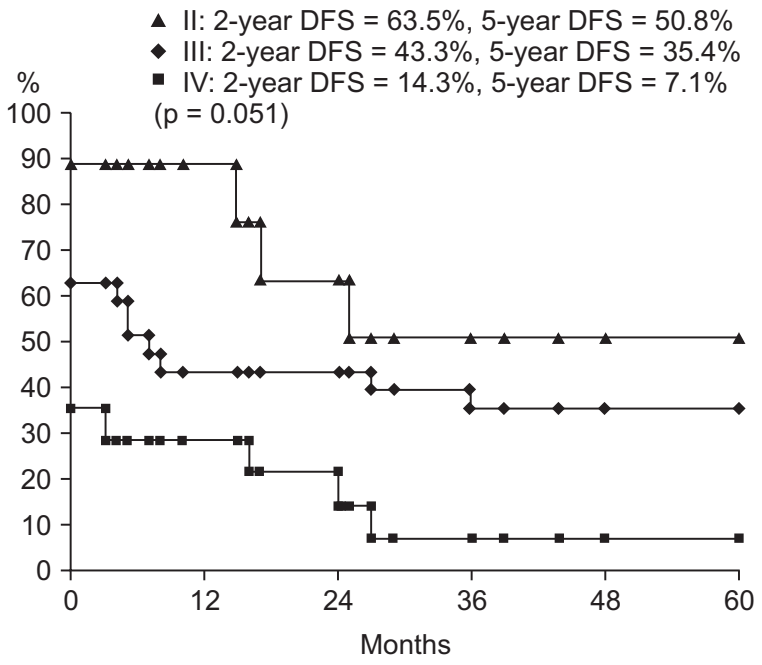

Fig. 4. Disease-free survival (DFS) curves according to clinical TNM stage.

Table 4. Univariate analysis of prognostic factors to OS and DFS

\begin{tabular}{|c|c|c|c|c|c|}
\hline Variable & No. (\%) & 5 -year OS (\%) & p-value & 5-year DFS (\%) & $p$-value \\
\hline Tumor grade & & & 0.051 & & NS \\
\hline Low & $12(27)$ & 65.6 & & 33.3 & \\
\hline High & $33(73)$ & 22.7 & & 22.4 & \\
\hline Clinical T stage & & & 0.089 & & NS \\
\hline 2 & $10(20)$ & 46.7 & & 45.7 & \\
\hline 3 & $30(60)$ & 43.3 & & 31.8 & \\
\hline 4 & $10(20)$ & 10.0 & & 10.0 & \\
\hline Clinical N stage & & & 0.087 & & 0.014 \\
\hline$(-)$ & $37(74)$ & 40.4 & & 38.4 & \\
\hline$(+)$ & $13(26)$ & 30.8 & & 7.7 & \\
\hline Clinical TNM stage & & & NS & & 0.015 \\
\hline II & $9(18)$ & 44.4 & & 50.8 & \\
\hline III & $27(54)$ & 40.7 & & 35.4 & \\
\hline IV & $14(28)$ & 26.8 & & 7.1 & \\
\hline Radiotherapy & & & 0.043 & & NS \\
\hline CCRT & $30(60)$ & 50.0 & & 39.1 & \\
\hline RT alone & $20(40)$ & 16.7 & & 16.4 & \\
\hline
\end{tabular}

OS, overall survival; DFS, disease-free survival; NS, no significance; CCRT, concurrent chemotherapy and radiotherapy; RT, radiotherapy. 
Table 5. Multivariate analysis of prognostic factors of OS

\begin{tabular}{ccc}
\hline \multirow{2}{*}{ Variable } & \multicolumn{2}{c}{ OS } \\
\cline { 2 - 3 } & & $95 \% \mathrm{Cl}$ \\
\hline Tumor grade & 1.00 & - \\
Low & 2.334 & $1.007-5.408$ \\
High & & $0.213-0.915$ \\
Radiotherapy & 0.442 & - \\
CCRT & 1.00 & \\
RT alone &
\end{tabular}

OS, overall survival; $\mathrm{HR}$, hazard ratio; $\mathrm{Cl}$, confidence interval; CCRT, concurrent chemotherapy and radiotherapy; RT, radiotherapy.

the small number of patients. There were only 50 patients selected to be analyzed in spite of relatively long 12-year follow-up period. It was not possible to access the acute and late toxicities caused by RT or chemotherapy, because patient data was collected retrospectively.

Survival rates seemed inferior to other results, although exact comparison is difficult, as other studies assessed treatment outcomes for different distribution of clinical stages $[9,11]$. Kotwal et al. [11] analyzed 169 patients and showed the treatment outcomes after radical RT in the form of EBRT or radical cystectomy. About a half of patients were in stage T3 or worse disease in radical RT group and the 5-year OS and disease-specific survival (DSS) was $34.6 \%$ and $56.8 \%$, respectively. A recent pooled analysis of RTOG reported 5-year OS of 57\% and 5-year DSS of 71\% in patients whose clinical stages were T3 or worse in 39\% [9]. In our study, $80 \%$ of patients were in clinical T3 or T4.

Patterns of failure after treatment were consistent with previously reported results. The majority of locoregional failures were in the bladder, and there were more noninvasive cases in the BC2001 study [17]. Presently, 11 out of 12 patients (91.7\%) had bladder recurrence, highlighting the importance of careful follow-up for bladder recurrence after bladder-preserving therapy. We also analyze the effect of concurrent chemotherapy on locoregional recurrence and distant metastasis. Synchronous cisplatin was reported to have a highly significant effect on pelvic recurrence and no effect on distant metastasis, whereas chemotherapy prior to RT had no effect on whether loco-regional recurrence or distant metastasis $[18,19]$. However, concurrent cisplatin was not effective on both locoregional recurrence $(p=0.137)$ and distant metastasis $(p=0.724)$ in our study. This may have reflected patient numbers; a larger number of patients may yield a satisfactory and accurate result.

For the evaluation of prognostic factors to survival rates, tumor grade was related to OS. It is already known to be the most important related to patient prognosis [20]. CCRT was another prognostic factor related to OS in multivariate analysis. Currently, although there are several combinations of concurrent or sequential chemotherapy with RT, concurrent chemotherapy with cisplatin is the most common combination to treat MIBC [21-23]. We also analyze clinical T stage and clinical N stage as well as clinical TNM stage for prognostic factors, respectively, because clinical T stage represents the depth of tumor invasion by itself and clinical $\mathrm{N}$ stage either negative or positive is able to determine clinical TNM stage, even in same clinical T stages.

During the follow-up period, all patients have their own bladder preserved, because no one underwent salvage cystectomy. However, it does not demonstrate successful treatment results. Eleven patients with local recurrences in bladder underwent repeated TURBT or palliative chemotherapy, instead of salvage cystectomy, due to old age or their poor medical conditions such as history of acute myocardial infarction or angina pectoris.

Bladder-preserving therapy in MIBC has several advantages including higher quality of life with comparable survival outcomes to radical cystectomy and is able to be adopted for patients with old age. For the bladder-preserving therapy including TURBT followed by RT, synchronous chemotherapy with RT would improves survival outcome. Tumor grade is also important prognostic factor for better survival outcomes. It is suggested that further prospective or multi-institutional studies are necessary for MIBC.

\section{Conflict of Interest}

No potential conflict of interest relevant to this article was reported.

\section{References}

1. Petrovich Z, Jozsef G, Brady LW. Radiotherapy for carcinoma of the bladder: a review. Am J Clin Oncol 2001;24:1-9.

2. Rodel C, Weiss C, Sauer R. Trimodality treatment and selective organ preservation for bladder cancer. J Clin Oncol 2006;24: 5536-44.

3. Chahal $R$, Sundaram SK, Iddenden $R$, Forman DF, Weston PM, Harrison SC. A study of the morbidity, mortality and long-term survival following radical cystectomy and radical radiotherapy in the treatment of invasive bladder cancer in Yorkshire. Eur Urol 2003;43:246-57. 
4. Mameghan H, Fisher R, Mameghan J, Brook S. Analysis of failure following definitive radiotherapy for invasive transitional cell carcinoma of the bladder. Int J Radiat Oncol Biol Phys 1995; 31:247-54.

5. Gospodarowicz MK, Hawkins NV, Rawlings GA, et al. Radical radiotherapy for muscle invasive transitional cell carcinoma of the bladder: failure analysis. J Urol 1989;142:1448-53.

6. Pollack A, Zagars GZ. Radiotherapy for stage T3b transitional cell carcinoma of the bladder. Semin Urol Oncol 1996;14:8695.

7. De Neve W, Lybeert ML, Goor C, Crommelin MA, Ribot JG. Radiotherapy for $\mathrm{T} 2$ and $\mathrm{T} 3$ carcinoma of the bladder: the influence of overall treatment time. Radiother Oncol 1995;36: 183-8.

8. Duncan W, Quilty PM. The results of a series of 963 patients with transitional cell carcinoma of the urinary bladder primarily treated by radical megavoltage X-ray therapy. Radiother Oncol 1986;7:299-310.

9. Mak RH, Hunt D, Shipley WU, et al. Long-term outcomes in patients with muscle-invasive bladder cancer after selective bladder-preserving combined-modality therapy: a pooled analysis of Radiation Therapy Oncology Group protocols 8802, 8903, 9506, 9706, 9906, and 0233. J Clin Oncol 2014;32:38019.

10. Munro NP, Sundaram SK, Weston PM, et al. A 10-year retrospective review of a nonrandomized cohort of 458 patients undergoing radical radiotherapy or cystectomy in Yorkshire, UK. Int J Radiat Oncol Biol Phys 2010;77:119-24.

11. Kotwal $S$, Choudhury $A$, Johnston $C$, Paul AB, Whelan P, Kiltie $A E$. Similar treatment outcomes for radical cystectomy and radical radiotherapy in invasive bladder cancer treated at a United Kingdom specialist treatment center. Int J Radiat Oncol Biol Phys 2008;70:456-63.

12. Madersbacher S, Hochreiter W, Burkhard F, et al. Radical cystectomy for bladder cancer today: a homogeneous series without neoadjuvant therapy. J Clin Oncol 2003;21:690-6.

13. Dalbagni G, Genega $E_{1}$ Hashibe $M$, et al. Cystectomy for bladder cancer: a contemporary series. J Urol 2001;165:1111-6. 14. Yu Jl, Park W, Oh DR, et al. Bladder preserving treatment in patients with muscle invasive bladder cancer. J Korean Soc Ther Radiol Oncol 2007;25:70-8.

15. Oken MM, Creech RH, Tormey DC, et al. Toxicity and response criteria of the Eastern Cooperative Oncology Group. Am J Clin Oncol 1982:5:649-55.

16. Eisenhauer EA, Therasse $P$, Bogaerts J, et al. New response evaluation criteria in solid tumours: revised RECIST guideline (version 1.1). Eur J Cancer 2009;45:228-47.

17. Halperin EC, Wazer DE, Perez CA, Brady LW. Perez and Brady's principles and practice of radiation oncology. 6th ed. Philadelphia, PA: Lippincott Williams \& Wilkins; 2013.

18. Coppin $\mathrm{CM}$, Gospodarowicz MK, James K, et al. Improved local control of invasive bladder cancer by concurrent cisplatin and preoperative or definitive radiation: the National Cancer Institute of Canada Clinical Trials Group. J Clin Oncol 1996;14: 2901-7.

19. Wallace DM, Raghavan D, Kelly KA, et al. Neo-adjuvant (pre-emptive) cisplatin therapy in invasive transitional cell carcinoma of the bladder. Br J Urol 1991;67:608-15.

20. Edge S, Byrd DR, Compton CC, Fritz AG, Greene FL, Trotti A. AJCC cancer staging manual. 7th ed. New York, NY: Springer; 2010.

21. Efstathiou JA, Spiegel DY, Shipley WU, et al. Long-term outcomes of selective bladder preservation by combinedmodality therapy for invasive bladder cancer: the MGH experience. Eur Urol 2012;61:705-11.

22. Shipley WU, Winter KA, Kaufman DS, et al. Phase III trial of neoadjuvant chemotherapy in patients with invasive bladder cancer treated with selective bladder preservation by combined radiation therapy and chemotherapy: initial results of Radiation Therapy Oncology Group 89-03. J Clin Oncol 1998;16:3576-83.

23. Shipley WU, Kaufman DS, Zehr E, et al. Selective bladder preservation by combined modality protocol treatment: longterm outcomes of 190 patients with invasive bladder cancer. Urology 2002;60:62-7. 